\title{
Optimal power efficient management scheme (OPEM): A new power efficient approach for mobile stations in two-tier and three-tier networks
}

\author{
Forough Shirin Abkenar and Akbar Ghaffarpour Rahbar* \\ Computer Networks Research Lab, Electrical Engineering Technologies Research Center, Sahand \\ University of Technology, Tabriz, Iran
}

\begin{abstract}
Establishing incoming calls plays an important role in multi-tier networks. In a multi-tier network, if a mobile station (MS) wants to move between different tiers, it has to register at its corresponding tier and deregister at current tier. The registration and deregistration procedures are implemented by network and radio signals. In addition, delivery of an incoming call to the corresponding tier, according to the type of call and the registration area (RA) of the mobile station, consumes power and energy. Many schemes like power efficient single registration (PSR), power efficient multiple registration (PMR) and efficient power management (EPM) have been introduced to reduce the power and energy consumptions of multi-tier networks. In this paper, we introduce a new approach to reduce the energy and power consumptions of multi-tier networks. This new approach is called the optimal power efficient management (OPEM) scheme that uses the advantages of both PMR and PSR in signaling concept. The OPEM scheme introduces a new, different and simple method to reduce the energy consumption of multi-tier networks, especially in two-tier and three-tier networks. The numerical results confirm the preference of the OPEM scheme.
\end{abstract}

Keywords: Two-tier networks, three-tier networks, location registration, paging, power consumption, optimal power efficient scheme

\section{Introduction}

The multi-tier network is a network that comprises convergence networks. In fact, convergence networks interwork together in the multi-tier network and each of them configures one tier. These interworked tiers are complementary of each other, i.e., each tier (i.e., network type) has the features or advantages that other tiers do not have. One conventional multi-tier network is interworked of cellular and wireless local area network (WLAN) networks (as a two-tier network). Figure 1 [1] shows the architecture of a two-tier network. As seen in Fig. 1, the two-tier network comprises high-tier and low-tier networks. In this two-tier network, the high-tier and the low-tier networks correspond to the cellular network and WLAN network, respectively. Another type of multi-tier networks is the three-tier network.

\footnotetext{
${ }^{*}$ Corresponding author: Akbar Ghaffarpour Rahbar, Computer Networks Research Lab, Electrical Engineering Technologies Research Center, Sahand University of Technology, Tabriz, Iran. E-mail: ghaffarpour@ sut.ac.ir.
} 


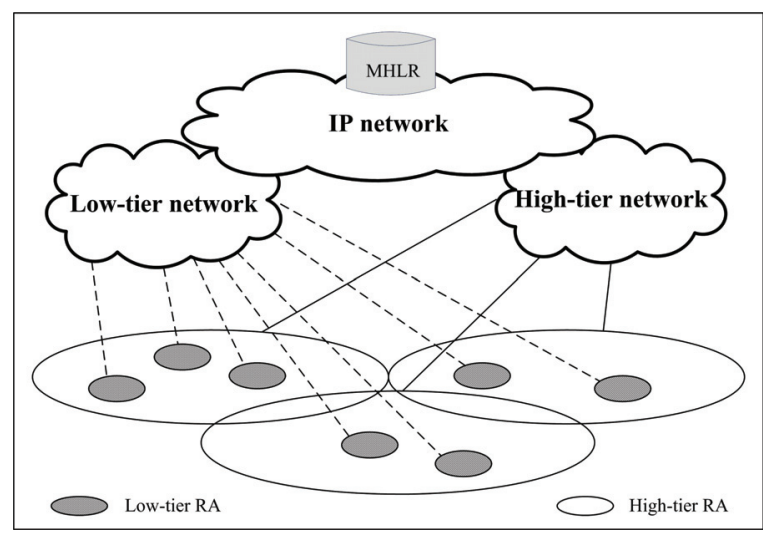

Fig. 1. Sample logical two-tier network architecture [1].

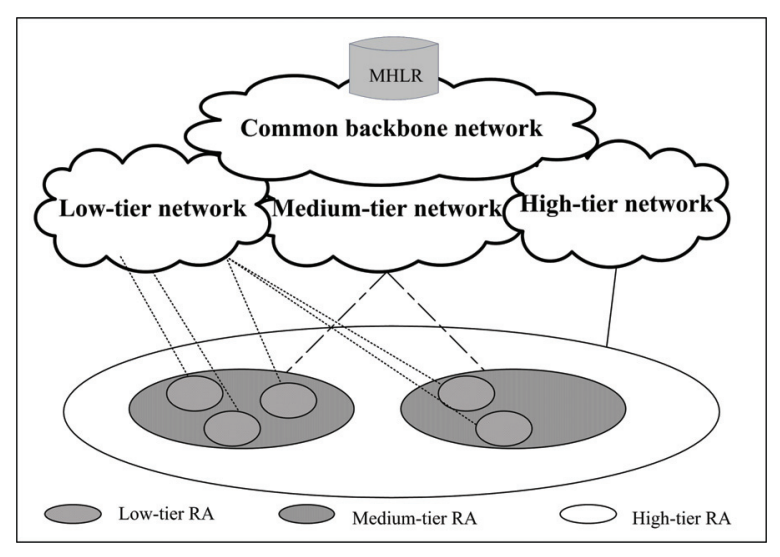

Fig. 2. Sample logical three-tier network architecture [2].

Figure 2 [2] shows the architecture of a three-tier network. As seen in Fig. 2, the three-tier network comprises low-tier, medium-tier and high-tier networks. As a new assumption, we can consider interworked of cellular/PON/WLAN networks as a three-tier network, where low-tier, medium-tier and high-tier networks correspond to WLAN, passive optical network (PON) and cellular network, respectively.

A cellular network is a type of wireless network, where an area is spliced into multiple cells. Each cell is serviced by its base station (BS). A BS has transmitters, receivers and a control unit that assigns different frequencies to each cell for avoiding interference. Each cell covers a circle environment, but a hexagon model is presented for a cellular network to simply calculate the differences between different cells and the assigned frequencies. The communications between mobile stations (MSs) is managed by the BS. The BSs are connected to a mobile telecommunication switching office (MTSO) that responses to establishing connections between MSs and the telecommunication center [3].

One constraint in local area networks (LANs) is mobility or scalability that limits the wired LAN performance. Hence, the wireless LANs (WLANs) have been introduced to solve the LAN constraints. WLAN is divided into two types: WLAN with infrastructure (that uses access points (APs) for communication) and WLAN without infrastructure (i.e., ad hoc networks). The WLAN involves advantages of LAN (such as high bandwidth provisioning, high security, high data rate), and mobility and scalability features [3].

A passive optical network (PON) is a type of optical networking that uses passive splitters, where the splitter can only broadcast the data coming from an input port to all output ports, but with less power. PON comprises an optical link terminal (OLT) and multiple optical network units (ONUs). The OLT is connected to a splitter and sends data to it. Then, the splitter splits the input data to all connected ONUs. The aim of PON is to provide data communication using optical technology at homes, buildings, offices because PON provides high transmission bandwidth and has good power consumption (less than wireless networks) [4].

In a multi-tier network, mobile stations (MSs) are mobile devices that move between different tiers. Each MS has to register at its corresponding tier to be enable to send and receive data. The most important issue in a multi-tier network is the amount of energy that the network consumes to deliver an incoming call to the appropriate tier. Many schemes have been introduced in this field. Two basic and important schemes are single registration (SR) and multi-registration (MR) schemes [5-7]. However, SR and MR are not energy efficient. Hence, the new methods have been introduced to optimize the energy consumption of multi-tier networks. Some types of these new optimized schemes are: efficient power 
management (EPM) and its developments [8-10], the introduced methods in [11-14], and the power efficient SR (PSR) and the power efficient MR (PMR) schemes [1,2].

In this paper, we introduce a new optimal approach that optimizes the energy consumption of multitier networks. The new approach is entitled optimal power efficient management scheme (OPEM). The OPEM inherits the advantages of the PMR and PSR schemes, i.e., low signaling load and turning off unnecessary radio interfaces. However, the delivery mechanism of incoming calls is different in OPEM.

The aim of OPEM is to reduce the energy consumption of multi-tier networks, specially two-tier and three-tier networks. In fact, OPEM uses the advantages of other schemes. In SR, an incoming call is delivered to a registered MS, irrespective of the type of the incoming call. In MR, the call is delivered to the MS corresponding to the type of the call (i.e., low-tier or high-tier call). However, in SR and MR, the low-tier and medium-tier interfaces are not turned off. Hence, the energy consumption of the network is high. In EPM, the low-tier and medium-tier interfaces are turned off and the high-tier network is responsible to inform and wake up the low-tier and medium-tier networks. Therefore, the energy consumption of the network can be decreased. The registration procedures in PSR and PMR are similar to SR and MR, respectively. However, when the low-tier, medium-tier or high-tier networks do not transmit/receive any data, these tiers can be turned off in PSR and PMR. In OPEM, MSs register at the RAs of all available access networks simultaneously like MR. In addition, when the low-tier, mediumtier or high-tier networks do not transmit/receive any data, these tiers can be turned off in OPEM like PSR or PMR. Note that the BSs and OLTs know which tiers are on or off. Hence, when an incoming call arrives, BSs or OLTs check wether the corresponding tier interface is on or off. If the interface is on, the incoming call is successfully delivered to it. However, if the interface is off, the OLT or BS wakes up the interface of the receiver tier and then delivers the incoming call to the corresponding tier. Hence, the incoming call is delivered to the corresponding tier irrespective of the type of data similar to SR. In fact, OPEM uses the advantages of available schemes and the awareness feature of BSs or OLTs to reduce the energy consumption of two-tier and three-tier networks.

In this paper, two-tier and three-tier networks are considered. In multi-tier networks, different tiers have to be complementary of each other. Note that a cellular network has high mobility support and large service coverage area. A passive optical network (PON) [15-17] covers smaller (bigger) area than cellular network (WLAN). On the other hand, PON has lower power consumption and higher bandwidth provisioning. In addition, WLAN has higher bandwidth provisioning than cellular network and mobility feature (while PON does not support mobility). Considering these characteristics, the wireless cellular network and local area network (WLAN) interwork together to configure a two-tier network. In an interworked cellular/WLAN network (as a two-tier network), the cellular network and WLAN correspond to high-tier and low-tier networks, respectively. On the other hand, cellular network, PON and WLAN interwork together to configure a three-tier network. In an interworked cellular/PON/WLAN network (as a three-tier network), the cellular network, PON and WLAN are considered as high-tier, medium-tier and low-tier networks, respectively.

Our contribution is to propose a new algorithm called optimal power efficient management (OPEM) that can significantly reduce the energy consumption of two-tier and three-tier networks. The OPEM is based on the awareness feature of BSs and OLTs to deliver incoming calls to corresponding tiers.

The rest of this paper is organized as follows. In Section 2, the multi-tier location management, the signaling procedure in OPEM and the energy consumtion in OPEM are represented. Section 3 consists of performance analysis and mathematical concepts of OPEM. In Section 4, the performance evaluation results are presented. Finally, Section 5 concludes the paper. 


\section{Optimal power efficient management scheme (OPEM)}

In this section, our proposed OPEM scheme is introduced. As mentioned in previous section, OPEM is the new scheme that has low signaling load and low power consumption.

Two-tier and three-tier networks have different structures. Figure 1 [1] and Fig. 2 [2] show the architecture of two-tier and three-tier networks, respectively. As seen in Fig. 1, a two-tier network comprises low-tier network (L), high-tier network $(\mathrm{H})$ and multi-tier home location register (MHLR). The MHLR manages the registration area (RA) information [1]. Moreover, as seen in Fig. 2, a three-tier network comprises low-tier network (L), medium-tier network (M), high-tier network (H) and MHLR [2].

\subsection{Multi-tier location management in OPEM}

Single registration (SR) and multi registration (MR) are the basic schemes in multi-tier location management. Each of these schemes involves low-tier interface (L), high-tier interface (H) and multi-tier home location register (MHLR) in a two-tier network [1]. In addition, SR and MR have low-tier interface $(\mathrm{L})$, medium-tier interface $(\mathrm{M})$, high-tier interface $(\mathrm{H})$ and multi-tier home location register (MHLR) in a three-tier network [2]. The low-tier network covers smaller area than the medium-tier network, and the medium tier network covers smaller area than the high-tier network. In the assumed two-tier network, the low-tier and high-tier networks correspond to WLAN and cellular network, respectively [1]. Moreover, in the assumed three-tier network, the low-tier, medium-tier and high-tier networks correspond to WLAN, PON and cellular network, respectively.

The details of existing schemes are expressed in Appendix A. Similar to MR, the MHLR manages the information of all interfaces in OPEM. Like PSR and PMR, the idle radio interfaces are off in OPEM.

The SR, PSR, MR, PMR, EPM and OPEM support quality of service (QoS) requirements of both voice and data calls [18]. The features of cellular network [19], PON [20-22] and WLAN [23,24] have been mentioned in the introduction section. Hence, if the network is three-tier and the user is fixed, the incoming data call is first delivered to PON, but if the user wants to move, the incoming data call is delivered to WLAN and the incoming voice call is delivered to an cellular network. In a two tier network, the incoming data call is delivered to WLAN and the incoming voice call is delivered to an cellular network.

Battery power consumption is an important issue in the SR and MR schemes, especially under the MR scheme. All of the interfaces have to work simultaneously in MR. Moreover, the low-tier and mediumtier interfaces consume more energy when these interfaces are idle (even more than when these interfaces are on). Hence, the power consumption of network is increased. A new approach has been introduced currently, called efficient power management (EPM) that is carried out in both two-tier and three-tier networks. When the WLAN interfaces or PON interfaces do not transmit and receive any data, they are turned off in the EPM scheme and woken up by the high-tier network. However, the signaling load in EPM is significant because paging for an incoming call at WLAN or PON is performed to all cells within an RA of the cellular network, which is generally larger than the RA of the WLAN and PON networks.

In [1,2], the advantages of all existing schemes (i.e., low signaling load feature in SR and MR and low power consumption feature in EPM) have been combined together and two new schemes entitled power efficient SR (called PSR) and power efficient MR (called PMR) have been introduced.

\subsection{Signaling procedure in OPEM}

We consider the signaling procedure for OPEM in two-tier and three-tier networks architecture (i.e., Fig. 1 [1] and Fig. 2 [2]). In OPEM, MSs register at the RAs of all available access networks simultaneously, like MR. Therefore, the MHLR in OPEM is similar to MHLR in MR. Note that, the turning 
on or off procedures can be done by a switch-off based approach like [25] and the QARWA mechanism [26] in wireless networks (i.e., cellular network and WLAN) and PON, respectively. Hence, when low-tier, medium-tier or high-tier networks do not transmit/receive any data, these tiers can be turned off in OPEM, like PSR or PMR. Since the BSs and OLTs know which tiers are on or off, the incoming call is delivered to corresponding tier irrespective of the type of data (like SR). Hence, OPEM uses the advantages of available schemes and can reduce the energy consumption of network in two-tier and three-tier networks.

\subsubsection{Signaling procedure of OPEM in two-tier networks}

An incoming call is delivered to an MS based on the relationship between the type of an incoming call and the location of an MS. The classification of this relationship in the two-tier network is expressed by the following cases [1]:

- Case(a): A high-tier call arrives at an MS when the MS is located in high-tier area.

- Case(b): A high-tier call arrives at an MS when the MS is located in low-tier area.

- Case(c): A low-tier call arrives at an MS when the MS is located in high-tier area.

- Case(d): A low-tier call arrives at an MS when the MS is located in low-tier area.

In OPEM, since existing BSs and OLT in different tiers transmit situation and control information among each other, the BSs and OLT know the on/off situation of each other. Note that when an incoming call arrives, the sender BS or OLT checks wether the interface of the receiver tier is on or off. If the interface of corresponding tier is on, the sender BS or OLT successfully delivers the incoming call to the corresponding interface. Otherwise, the sender BS or OLT wakes up the interface of corresponding tier, and then delivers the incoming call to it. In fact, MHLR in OPEM has information of all tiers (like MR or PMR) and the call is delivered to an on interface (like SR or PSR that the incoming call is only delivered to the registered interface). In different cases, we have the following issues in the two-tier network under OPEM:

- In cases (a) and (c), the low-tier interfaces are off.

- In cases (b) and (d), the high-tier interfaces are off.

- In cases (a) and (c), the incoming call is delivered to the tier that its interface is on (i.e., high-tier), irrespective of the type of the incoming call. Hence, the paging is performed by high-tier RAs.

- In cases (b) and (d), the incoming call is delivered to the tier that its interface is on (i.e., low-tier). Hence, the paging is performed by high-tier RAs.

\subsubsection{Signaling procedure of OPEM in three-tier networks}

As mentioned before, an incoming call is delivered to an MS based on the relationship between the type of the incoming call and the location of the MS. The classification of this relationship in the threetier network is expressed by the following cases:

- Case(a-3): A high-tier call arrives at an MS when the MS is located in high-tier area.

- Case(b-3): A high-tier call arrives at an MS when the MS is located in medium-tier area.

- Case(c-3): A high-tier call arrives at an MS when the MS is located in low-tier area.

- Case(d-3): A medium-tier call arrives at an MS when the MS is located in high-tier area.

- Case(e-3): A medium-tier call arrives at an MS when the MS is located in medium-tier area.

- Case(f-3): A medium-tier call arrives at an MS when the MS is located in low-tier area.

- Case(g-3): A low-tier call arrives at an MS when the MS is located in high-tier area.

- Case(h-3): A low-tier call arrives at an MS when the MS is located in medium-tier area.

- Case(i-3): A low-tier call arrives at an MS when the MS is located in low-tier area. 
In addition, in different cases, we have the following issues in the three-tier network under OPEM:

- In cases (a), (d) and (g), the low-tier and medium-tier interfaces are off.

- In cases (b), (e) and (h), the high-tier and low-tier interfaces are off.

- In cases (c), (f) and (i), the high-tier and medium-tier interfaces are off.

- In cases (a), (d) and (g), the incoming call is delivered to the tier that its interface is on (i.e., hightier), irrespective of the type of the incoming call. Hence, the paging is performed by high-tier RAs.

- In cases (b), (e) and (h), the incoming call is delivered to the tier that its interface is on (i.e., mediumtier). Hence, the paging is performed by medium-tier RAs.

- In cases (c), (f) and (i), the incoming call is delivered to the tier that its interface is on (i.e., low-tier). Hence, the paging is performed by low-tier RAs.

\subsection{Energy saving feature of OPEM compared to other techniques}

In the SR schemes, an MS can register at only one RA and an incoming call is delivered to the registered MS. In addition, the low-tier and medium-tier interfaces are on in SR. The signaling and call delivering procedures of PSR are similar to SR, but in PSR the low-tier and medium-tier interfaces are off. In addition, an MS can simultaneously register at only one RA. Hence, the registration of each interface, separately, consumes more energy. In MR, an MS can simultaneously register at all available RAs. In addition, the incoming call is delivered to the corresponding tier, according to the call type. However, the low-tier and medium-tier interfaces are on in MR. The signaling and call delivering procedures of PMR are similar to MR, but in PMR the high-tier, low-tier and medium-tier interfaces can be off. Moreover, when a call arrives, the type of call is considered and the call has to be delivered to the tier with the same type. Hence, a call may be delivered to a tier that its interfaces are off. Therefore, the call has to be delivered to the appropriate tier, again. This procedure consumes some amount of energy. In EPM, the low-tier and medium-tier interfaces are off and the high-tier responses to inform the low-tier and medium-tier networks that an incoming call has been arrived. This process significantly reduces the energy consumption.

Finally, an MS can register at all available RAs in OPEM. In addition, the high-tier, low-tier and medium-tier interfaces can be off. However, the incoming call is delivered to the corresponding tier, irrespective of the type of the incoming call. In PSR or PMR, the tiers that their interfaces are off are woken up periodically. But in OPEM, different tiers can wake up each other. In fact, since existing BSs and OLT in different tiers transmit situation and control information between each other, the BSs and OLT know the on/off situation of each other. Hence, when a call arrives, if the interface of corresponding tier is on, the incoming call is successfully delivered to the tier. However, if the interface of the corresponding tier is off, the sender BS or OLT first wakes up the receiver BS or OLT. Then, the sender BS delivers the incoming call to the receiver BS or OLT. Therefore, the energy consumption is significantly reduced.

\section{Performance analysis}

In this section, the performance of OPEM is analyzed from the signaling load and energy consumption aspects in two-tier networks and from the energy consumption aspect in three-tier networks. Hence, the mathematical modeling of mobility and traffic characteristics of mobile users is expressed to analyze the performance of power efficient multi-tier location management, mean value analysis based on Poisson arrivals, and exponential distribution for simplicity of calculations. 


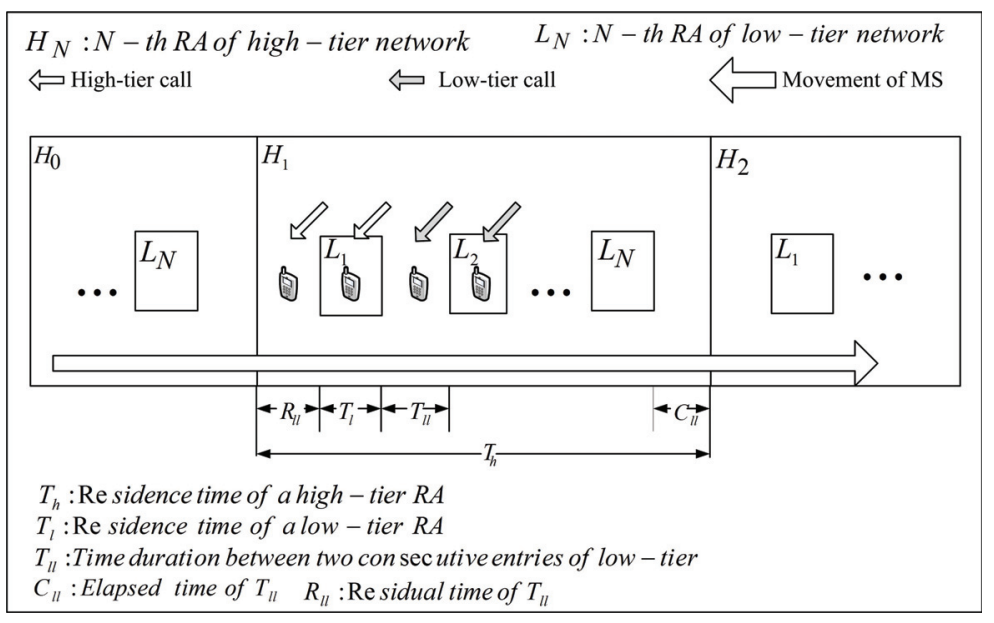

Fig. 3. Timing diagram for two-tier network [1].

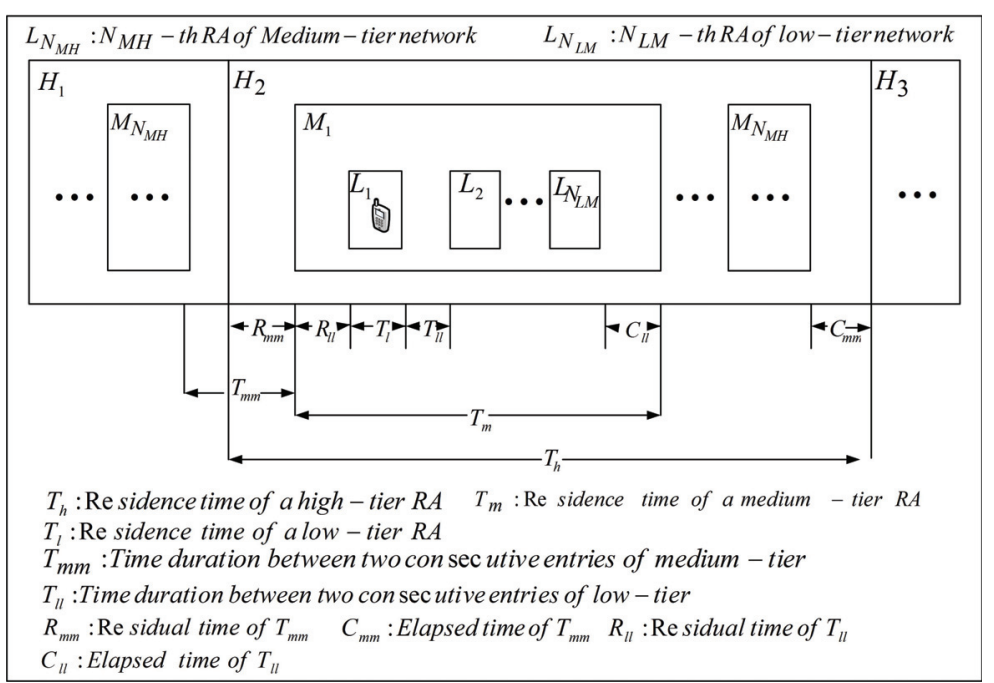

Fig. 4. Timing diagram for three-tier network [2].

\subsection{Mobility and traffic modelling}

Figure 3 [1] shows a timing diagram for performance analysis of two-tier location management schemes. In this figure, an MS moves from (a) to (d) as time goes on. The states (a)-(d) are the same cases (a)-(d) that were mentioned in Section 2. In addition, Fig. 4 [2] shows a timing diagram for the performance analysis of the three-tier location management schemes. According to these timing diagrams, there are some mathematical assumptions for both two-tier and three-tier location management schemes as follows [1,2]:

- The total number of low-tier RAs crossed by an MS during the residence time of a high-tier RA is $N$ (in two-tier scheme) [1].

- The total number of low-tier RAs crossed by an MS during the residence time of a medium-tier RA is $N_{L H}$ [2]. 
Table 1

The number of signaling messages for location registration

\begin{tabular}{llccc}
\hline Scheme & Signaling & Case(1) & Case(2) & Case(3) \\
\hline SR & Network & 4 & 4 & 4 \\
SR & Radio & 2 & 2 & 2 \\
PSR & Network & 4 & 4 & 4 \\
PSR & Radio & 2 & 2 & 2 \\
MR & Network & 4 & 4 & 0 \\
MR & Radio & 2 & 2 & 0 \\
PMR & Network & 4 & 4 & 0 \\
PMR & Radio & 2 & 2 & 0 \\
EPM & Network & 4 & 0 & 0 \\
EPM & Radio & 2 & 0 & 0 \\
OPEM & Network & 4 & 4 & 0 \\
OPEM & Radio & 2 & 2 & 0 \\
\hline
\end{tabular}

- The total number of medium-tier RAs crossed by an MS during the residence time of a high-tier RA is $N_{M H}$ [2].

- Each low-tier RA consists of $N_{C L}$ low-tier cells [1].

- Each high-tier RA consists of $N_{C H}$ high-tier cells [1].

- Low-tier call arrivals to an MS follow Poisson arrivals with mean arrival rate parameter $\lambda_{L}[1,2]$.

- Medium-tier call arrivals to an MS follow Poisson arrival with mean arrival rate parameter $\lambda_{M}$ [2].

- High-tier call arrivals to an MS follow Poisson arrival with mean arrival rate parameter $\lambda_{H}[1,2]$.

- Low-tier call durations follow Exponential distribution with mean service rate parameter $\mu_{L}[1,2]$.

- Medium-tier call durations follow Exponential distribution with mean service rate parameter $\mu_{M}[2]$.

- High-tier call durations follow Exponential distribution with mean service rate parameter $\mu_{H}[1,2]$.

- Residence time of a low-tier RA, $T_{l}$, follows Exponential distribution with mean service rate parameter $\mu_{l}[1,2]$.

- Residence time of a high-tier RA, $T_{h}$, follows Exponential distribution with mean service rate parameter $\mu_{h}[1]$.

- The time duration between two consecutive entries of low-tier RA, $T_{l l}$, follows Exponential distribution with mean service rate parameter $\mu_{l l}[1]$.

- The time duration between two consecutive entries of medium-tier RA, $T_{m m}$, follows Exponential distribution with mean service rate parameter $\mu_{m m}$ [2].

First, we calculate radio, network and total signallings of OPEM scheme in two-tier networks. In addition, we obtain the energy consumption of OPEM in both two-tier and three-tier networks. For this, we use the exiting equations in $[1,2]$ to calculate the signaling loads and energy consumptions of the OPEM scheme.

\subsection{Signaling load}

In this section, we calculate the total signaling load for OPEM in two-tier location management based on the equations presented in [1]. For signaling load analysis, the following notations are defined [1]:

- $h_{\text {reg }}$ : Unit signaling load of transmitting a registration message at network [1].

- $h_{\text {pag }}$ : Unit signaling load of transmitting a paging message at network [1].

- $g_{\text {reg }}$ : Unit signaling load of transmitting a registration message at radio interface [1].

- $g_{\text {pag }}$ : Unit signaling load of transmitting a paging message at radio interface [1]. 
Table 2

The number of signaling messages for paging

\begin{tabular}{llcccc}
\hline Scheme & Signaling & Case(a) & Case(b) & Case(c) & Case(d) \\
\hline SR & Network & 2 & 2 & 2 & 2 \\
SR & Radio & $N_{C H}+1$ & $N_{C L}+1$ & $N_{C H}+1$ & $N_{C L}+1$ \\
PSR & Network & 2 & 2 & 2 & 2 \\
PSR & Radio & $N_{C H}+1$ & $N_{C L}+1$ & $N_{C H}+1$ & $N_{C L}+1$ \\
MR & Network & 2 & 4 & 4 & 2 \\
MR & Radio & $N_{C H}+1$ & $N_{C H}+N_{C L}+1$ & $N_{C H}+N_{C L}+1$ & $N_{C L}+1$ \\
PMR & Network & 2 & 4 & 4 & 2 \\
PMR & Radio & $N_{C H}+1$ & $N_{C H}+N_{C L}+1$ & $N_{C H}+N_{C L}+1$ & $N_{C L}+1$ \\
EPM & Network & 2 & 2 & 2 & 2 \\
EPM & Radio & $N_{C H}+1$ & $N_{C H}+1$ & $N_{C H}+1$ & $N_{C H}+1$ \\
OPEM & Network & 2 & 2 & 2 & 2 \\
OPEM & Radio & $N_{C H}+1$ & $N_{C L}+1$ & $N_{C H}+1$ & $N_{C L}+1$ \\
\hline
\end{tabular}

Table 3 Parameter values $[1,2]$

\begin{tabular}{lr}
\hline$\lambda_{L}$ & 10 \\
\hline$\mu_{L}$ & 100 \\
$\lambda_{M}$ & 6 \\
$\mu_{M}$ & 60 \\
$\lambda_{H}$ & 3 \\
$\mu_{H}$ & 30 \\
$\mu_{l}$ & 10 \\
$\mu_{l l}$ & 10 \\
$\mu_{m m}$ & 2 \\
$h_{\text {reg }}$ & 1 \\
$h_{\text {pag }}$ & 1 \\
$g_{r e g}$ & 1 \\
$g_{\text {pag }}$ & 1 \\
$N_{C H}$ & 50 \\
$N_{C L}$ & 5 \\
$N_{L H}$ & 10 \\
$N_{M H}$ & 5 \\
\hline
\end{tabular}

Tables 1 and 2 show the number of signaling messages for location registration and paging, respectively, at network and radio interfaces, based on the signaling procedures in Section 2. In Table 1, Cases(1)-(3) represent the following three cases where location registrations occur [1]:

- Case(1): An MS moves between high-tier RAs [1].

- Case(2): An MS moves into a low-tier RA [1].

- Case(3): An MS moves out of a low-tier RA [1].

Using the above notations, network signaling load for OPEM registration is obtained using Table 1 and Eq. (1) as following:

$$
N S_{O P E M}^{r e g}=\frac{\left(4 h_{r e g}+4 N h_{r e g}\right)}{\theta_{T}^{2}}=\frac{4(1+N) h_{r e g}}{\theta_{T}^{2}} .
$$

where $\theta_{i}^{j}$ shows the mean time duration type $i$ in the $j$-tier location management schemes. The relations between these parameters have been expressed in $[1,2]$.

Similarly, radio signaling load for registration is obtained using Table 1 and Eq. (2) as follows:

$$
R S_{O P E M}^{r e g}=\frac{\left(2 g_{r e g}+2 N g_{r e g}\right)}{\theta_{T}^{2}}=\frac{2(1+N) g_{r e g}}{\theta_{T}^{2}} .
$$

For paging, network signaling load is obtained by using Table 2 and Eq. (3) as follows:

$$
N S_{O P E M}^{p a g}=2 h_{\text {pag }} \lambda_{H} \pi_{H}^{2}+2 h_{\text {pag }} \lambda_{H} \pi_{L}^{2}+2 h_{\text {pag }} \lambda_{L} \pi_{H}^{2}+2 h_{\text {pag }} \lambda_{L} \pi_{L}^{2}=2 h_{\text {pag }}\left(\lambda_{H}+\lambda_{L}\right) .
$$

where $\pi_{i}^{j}$ represents the steady state probability of an MS at tier $i$ in the $j$-tier location management scheme. The relation between these parameters has been expressed in $[1,2]$.

Radio signaling load for paging is obtained by using Table 2 and Eq. (4) as follows:

$$
\begin{aligned}
R S_{O P E M}^{\text {pag }}= & \left(N_{C H}+1\right) g_{\text {pag }} \lambda_{H} \pi_{H}^{2}+\left(N_{C L}+1\right) g_{\text {pag }} \lambda_{H} \pi_{L}^{2}+\left(N_{C H}+1\right) g_{\text {pag }} \lambda_{L} \pi_{H}^{2} \\
& +\left(N_{C L}+1\right) g_{\text {pag }} \lambda_{L} \pi_{L}^{2}=\left(N_{C H}+1\right) \pi_{H}^{2} g_{p a g}\left(\lambda_{H}+\lambda_{L}\right) \\
& +\left(N_{C L}+1\right) \pi_{L}^{2} g_{p a g}\left(\lambda_{H}+\lambda_{L}\right) .
\end{aligned}
$$


Table 4

Power consumption

\begin{tabular}{lccccc}
\hline$P_{\text {active }}^{H}$ & $P_{\text {idle }}^{H}$ & $P_{\text {active }}^{M}$ & $P_{\text {idle }}^{M}$ & $P_{\text {active }}^{L}$ & $P_{\text {idle }}^{L}$ \\
\hline $1.254 \mathrm{~W}$ & $0.125 \mathrm{~W}$ & $0.8 \mathrm{~W}$ & $0.1 \mathrm{~W}$ & $1.65 \mathrm{~W}$ & $1.15 \mathrm{~W}$ \\
\hline
\end{tabular}

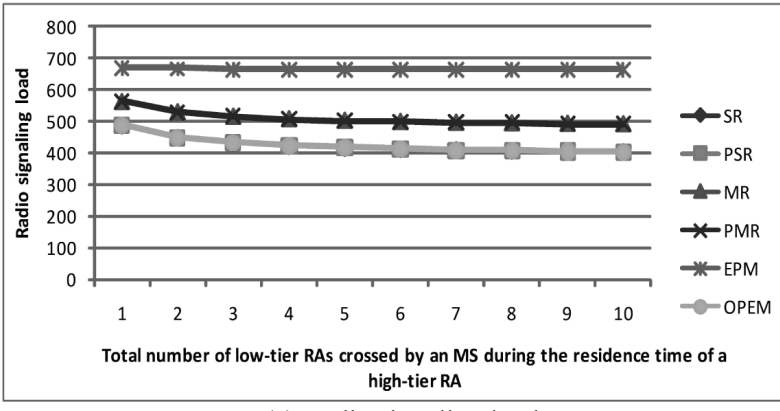

(a) Radio signaling load

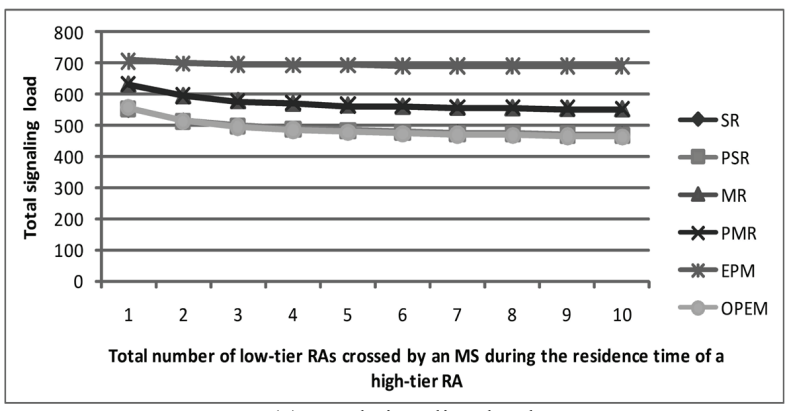

(c) Total signaling load

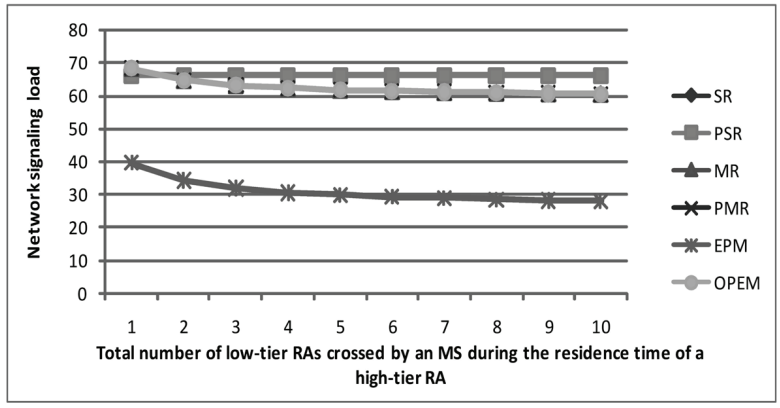

(b) Network signaling load

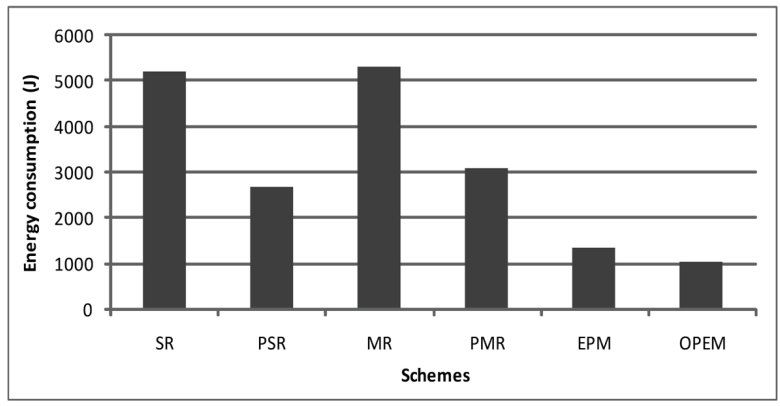

(d) Energy consumption

Fig. 5. Signaling loads and energy consumption for varying the values of the total number of low-tier RAs crossed by an MS during the residence time of a high-tier RA, $N$.

\subsection{Energy consumption}

In this section, we calculate the energy consumption of both two-tier and three-tier location management schemes. Define $E_{i}^{j}$ to represent the energy consumption of Scheme $i$ (where $i=\mathrm{SR}$, PSR, MR, PMR, EPM, OPEM) per unit time for the $j$-tier location management scheme. Energy consumptions of two and three-tiers schemes have been defined in [1,2]. Hence, the energy consumption of OPEM in two-tier and three tier networks are obtained by using Eqs (5) and (6) as following:

$$
\begin{aligned}
E_{\text {OPEM }}^{2}= & \left(\frac{\lambda_{H}}{\mu_{H}}+\frac{\lambda_{L}}{\mu_{L}}\right) \pi_{H}^{2} P_{\text {active }}^{H}+\left(\frac{\lambda_{H}}{\mu_{H}}+\frac{\lambda_{L}}{\mu_{L}}\right) \pi_{L}^{2} P_{\text {active }}^{L} \\
E_{\text {OPEM }}^{3}= & \left(\frac{\lambda_{H}}{\mu_{H}}+\frac{\lambda_{M}}{\mu_{M}}+\frac{\lambda_{L}}{\mu_{L}}\right) \pi_{H}^{3} P_{\text {active }}^{H}+\left(\frac{\lambda_{H}}{\mu_{H}}+\frac{\lambda_{M}}{\mu_{M}}+\frac{\lambda_{L}}{\mu_{L}}\right) \pi_{M}^{3} P_{\text {active }}^{M} \\
& +\left(\frac{\lambda_{H}}{\mu_{H}}+\frac{\lambda_{M}}{\mu_{M}}+\frac{\lambda_{L}}{\mu_{L}}\right) \pi_{L}^{3} P_{\text {active }}^{L},
\end{aligned}
$$




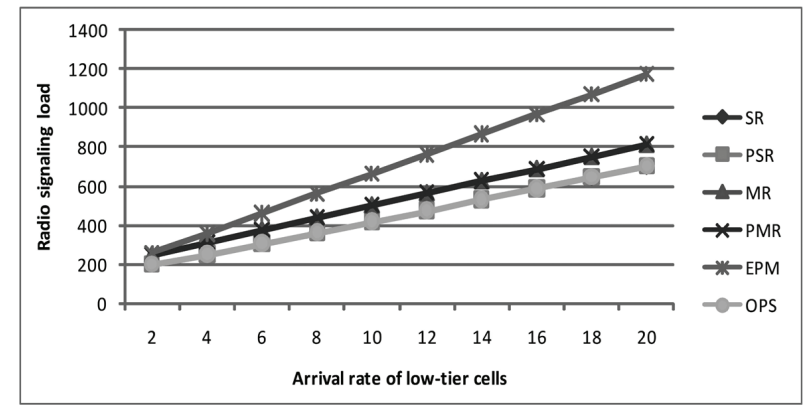

(a) Radio signaling load

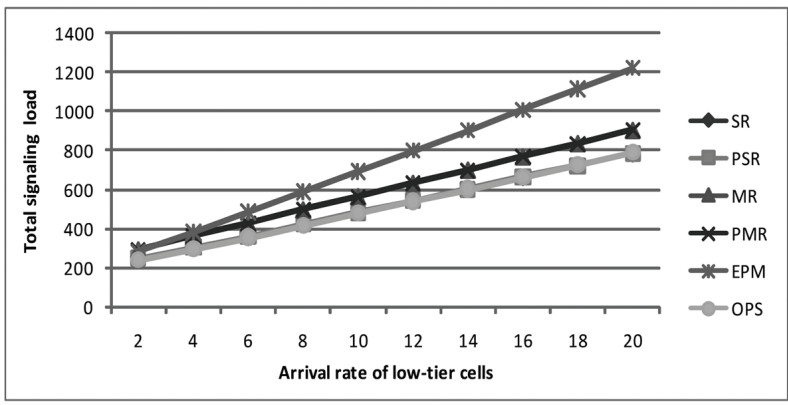

(c) Total signaling load

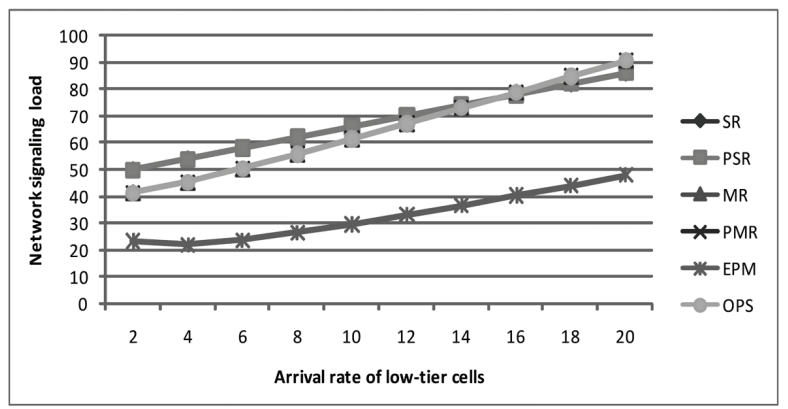

(b) Network signaling load

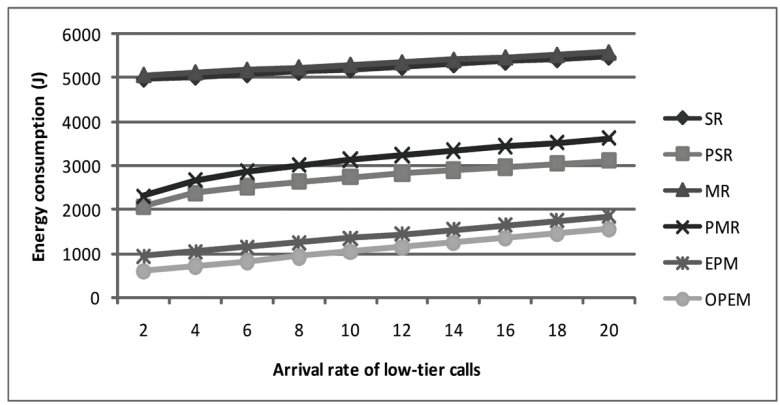

(d) Energy consumption

Fig. 6. Energy consumption for varying the values of arrival rate of low-tier, $\lambda_{L}$.

\section{Numerical results}

In this section, we uses the information of Table $3[1,2]$ and our Table 4 for our performance evaluation. In addition, the represented equations in [1,2], and our equations in Eqs (1)-(6).

\subsection{Numerical results of the two-tier network}

Figures 5 and 6 show the numerical results of the two-tier network. In Fig. 5, the total number of low-tier RAs $(N)$ has changed. As seen in Figs 5(a)-(c), the radio signaling and total signaling of OPEM is less than other schemes, but the network signaling of OPEM is more than EPM. This is because the radio signaling of OPEM follows the SR or the PSR schemes that only page with on interfaces. However, the network signaling of OPEM follows the MR or the PMR schemes that have less signaling load than SR and PSR. Note that in EPM, the network paging is performed only with the high-tier network.

As it can be observed in Fig. 5(d), OPEM has reduced the energy consumption significantly. The energy consumption of OPEM is very low, even much lower than the best and the efficient scheme such as EPM. In Fig. 6, the value of arrival rate of low-tier calls (i.e., $\lambda_{L}$ ) has changed. As Figs 6(a)(c) show, when the arrival rate of low-tier call is increased, all schemes have to do registration and paging procedures more. Hence, the signaling load in both registration and paging phases is increased. Therefore, network and radio signaling have an ascending process. Since the total signal load is based on the network and radio signaling loads, the total signaling load has an ascending procedure.

In addition, as it can be seen in Fig. 6(d), in this condition, the OPEM has the best performance in energy consumption like the result of Fig. 5(d). Moreover, by increasing the arrival rates of low-tier calls, the amount of energy consumption is increased linearly. 


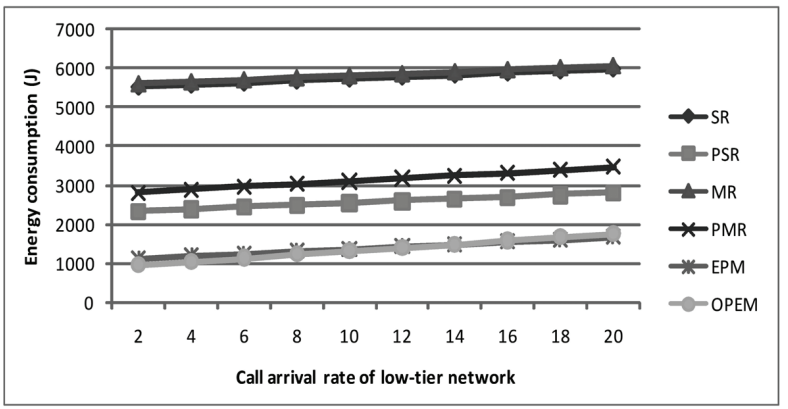

(a) Low-tier call arrival rate

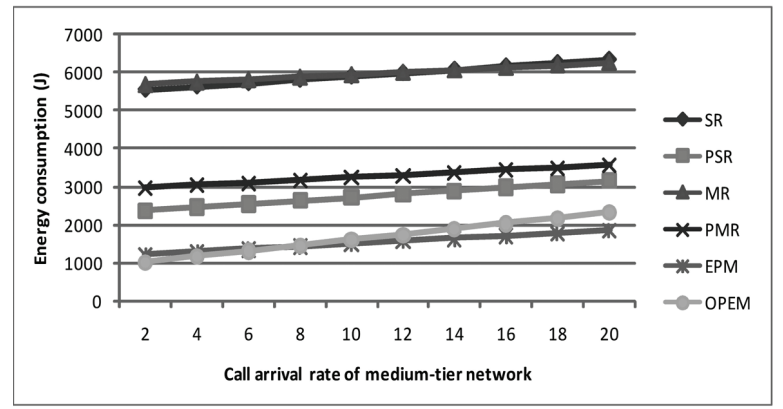

(b) Medium-tier call arrival rate

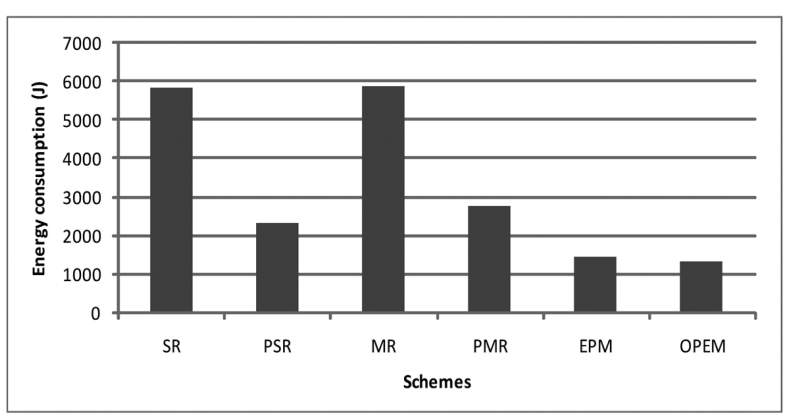

(c) $N_{L M}$ and $N_{M H}$

Fig. 7. Energy consumption for varying the values of arrival rates of low-tier and medium-tier and the total numbers of low-tier and medium-tier RAs, $\lambda_{L}, \lambda_{M}, N_{L M}$ and $N_{M H}$.

\subsection{Numerical results of three-tier network}

Figure 7 shows the energy consumption of different schemes in the three-tier network. In Figs 7(a) and (b), the values of arrival rates of low-tier and medium-tier have changed, respectively. In addition, in Fig. 7(c), the total numbers of low-tier and medium-tier calls have changed. As seen in both Figs 7(a) and (b), the OPEM has a performance like EPM, roughly. Moreover, as seen in Fig. 7(c), OPEM has the best energy consumption, even better than EPM. Note that when the arrival rates of low-tier or medium-tier calls are increased, the network has to consume more energy to deliver the incoming call to appropriate tiers. Hence, the energy consumption of all schemes is increased linearly. Note that the unit time of these analyzes is hour that has been converted to seconds in all numerical scenarios.

\section{Conclusions}

In this paper, a new multi-tier location management scheme, called OPEM, has been introduced. In OPEM, unused interfaces are turned off. However, the main idea of OPEM is the knowledge of different tiers BSs from each other. In fact, all tiers BSs know which interfaces are off or on. When a call arrives, the call is delivered to the tier with on interfaces. The OPEM reduces the amount of energy consumption in two-tier networks, even more than the scheme with the best energy efficiency, i.e., EPM. However, in the three-tier network, the performances of OPEM and EPM are similar to each other. Even, when the arrival rates of medium-tiers is increased, EPM has better result than OPEM. However, when the total numbers of low-tier and medium-tier calls are increased, the OPEM has better energy consumption than all schemes, especially EPM. 


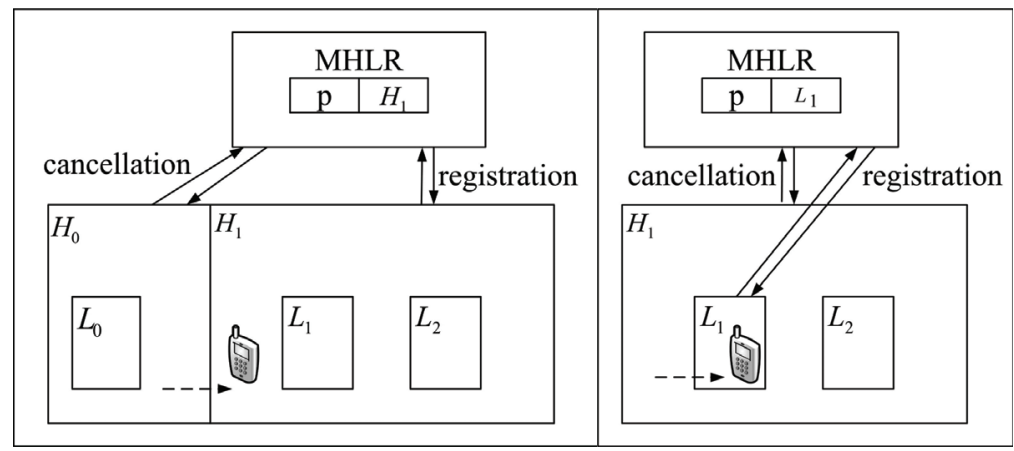

(a)

(b)

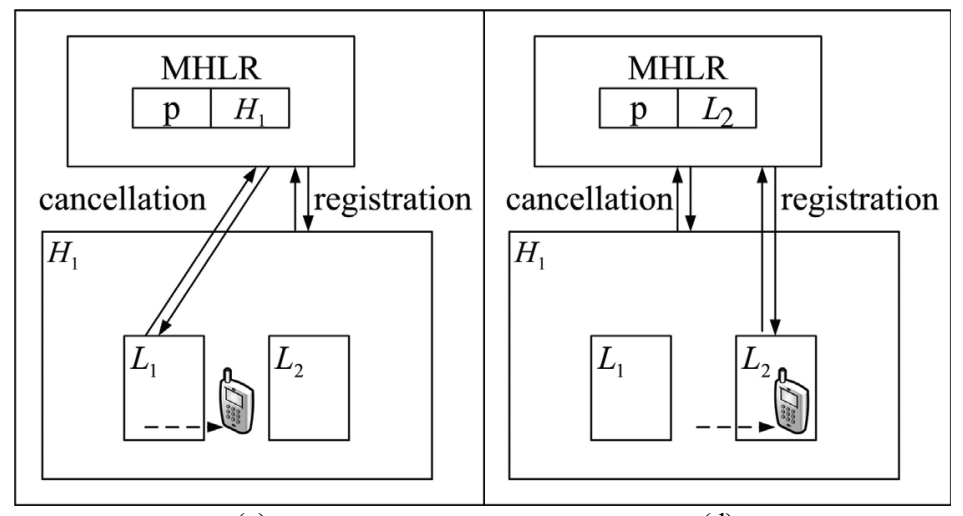

(c)

(d)

Fig. 8. Single registration (SR) scheme [1].

\section{Appendix Detailing the available schemes}

\section{Registration mechanism of SR, PSR, MR, PMR and EPM in two-tier networks}

In this section, the registration mechanism of SR, PSR, MR, PMR and EPM schemes are described as:

- SR: In SR, MSs register at the registration area (RA) of only one of the available access networks. Figure 8 [1] depicts the SR scheme in a two-tier network architecture. As seen in Fig. 8(a), the MS $p$ is located at high-tier $H_{0}$ and registered at MHLR. The MS decides to move into another high-tier area $H_{1}$. Hence, the MS should deregister $H_{0}$ at MHLR and register $H_{1}$ at MHLR. In Fig. 8(b), the MS moves to low-tier $L_{1}$, hence it has to deregister $H_{1}$ at MHLR and register $L_{1}$ at MHLR. In continuance, according to Fig. 8(c), the MS exits from $L_{1}$ and enters to $H_{1}$. In the next step, according to Fig. 8(d), the MS enters to new low-tier $L_{2}$. Therefore, it should do deregistration and registration operations again at MHLR [1].

- MR: In MR, an MS simultaneously registers at the RAs of all available networks at MHLR. Figure 9 [1] shows the MR scheme in a two-tier network architecture. As seen in Fig. 9(a) [1], an MS $p$ moves from a high-tier $H_{0}$ into another high-tier $H_{1}$. Hence, the MS should deregister $H_{0}$ at MHLR and register $H_{1}$ at MHLR. After that, according to Fig. 9(b), MS moves to a low-tier area $L_{1}$. Unlike the SR scheme, it is enough to deregister previous low-tier at MHLR and register at low-tier segment of MHLR without any high-tier (de)registration. Then, as seen in Fig. 9(c), the 


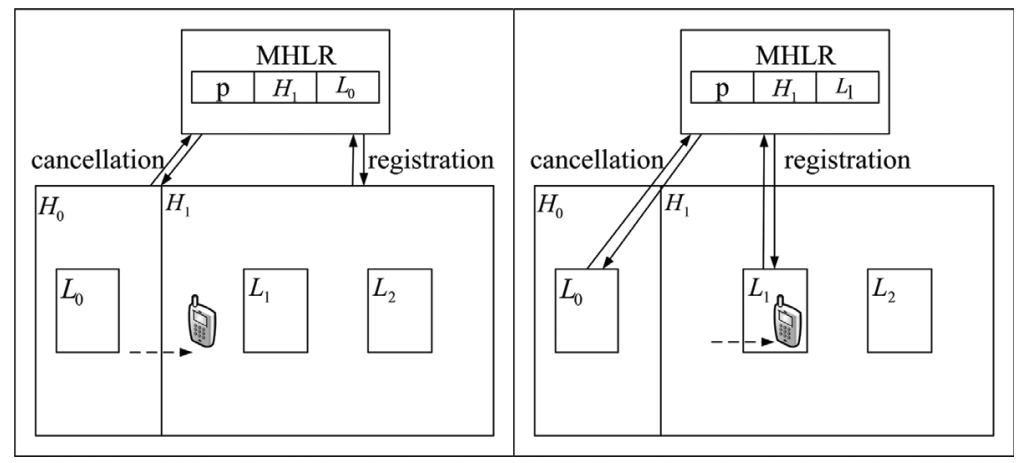

(a)

(b)

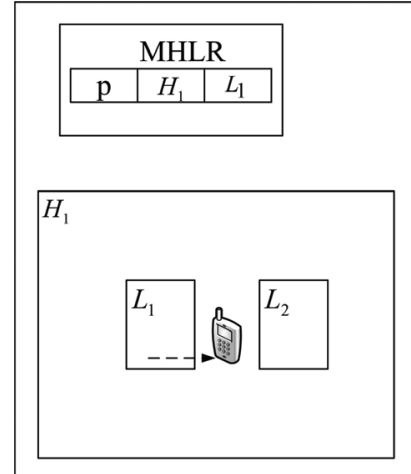

(c)

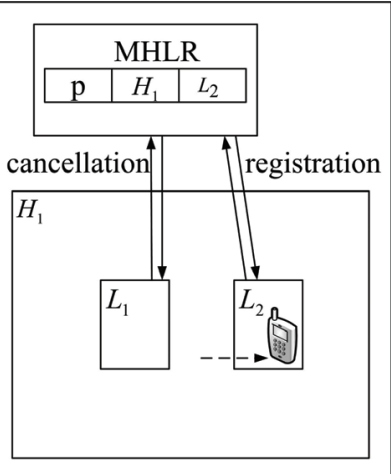

(d)

Fig. 9. Multi registration (MR) scheme [1].

MS moves into new low-tier area $L_{2}$. In SR, the MS had to (de)register high-tier area at MHLR, but in MR no (de)registration operations are performed in this step. This is because the registration information of $H_{1}$ has been stored in MHLR. Finally, the MS enters to new low-tier $L_{2}$. Therefore, according to Fig. 9(d), it should do deregistration and registration operations, only in the low-tier RA [1].

- PSR: The location registration and paging procedures in PSR are similar to SR so that only the low-tier, medium-tier or high-tier interfaces can be off. In fact, PSR improves power and energy consumption [1].

- PMR: The location registration and paging procedures in PMR are similar to MR so that only the low-tier, medium-tier or high-tier interfaces can be off in PMR. In fact, PMR improves power and energy consumption [1].

- EPM: In the EPM scheme, the low-tier interfaces are off and the registration is performed by the high-tier network [8-10].

\section{Signaling mechanism of SR, PSR, MR, PMR and EPM in two-tier networks}

In this section, the signaling mechanism of SR, PSR, MR, PMR and EPM schemes are described as:

- SR: In SR, an incoming call is delivered to the registered RA irrespective of the type of the incoming call (i.e., low-tier call, middle-tier call or high-tier call). In all mentioned cases (a)-(d)(see Section 2), calls are delivered to the corresponding MS [1]. 
- MR: In MR, an incoming call is first delivered to the corresponding tier. In case (a), the high-tier call is delivered to the high-tier RA. In case (b), a high-tier call is first delivered to the high-tier RA, but since the MS is not within the high-tier RA, there is no response from the high-tier RA. Therefore, the incoming call is delivered to the low-tier RA. In case (c), the low-tier call is first delivered to the low-tier RA, but the MS is not within the low-tier RA. Hence, the call is delivered to high-tier RA. In case (d), the low-tier call is successfully delivered to the low-tier RA.

- EPM: In EPM, the low-tier interfaces are turned off. Hence, the paging is performed by high-tier RAs. In cases (a) and (c), the incoming calls are successfully delivered to high-tier RAs. However, in cases (b) and (d), the incoming call is first delivered to the high-tier RAs. But, since the MSs are not within the high-tier RAs. Hence, the high-tier network wakes up the low-tier interfaces, and then incoming calls are delivered to the low-tier network [1].

- PSR: Paging and registration in PSR are similar to SR, but the radio interfaces of the network that are not registered in MHLR are turned off for power saving, and waken up periodically to check the entrance into the low-tier or middle-tier RAs [1].

- PMR: Paging and registration in PMR are similar to MR, but when an MS is located in a high-tier or medium-tier RA, the low-tier radio interfaces are turned off to reduce the energy consumption. Similarly, when an MS is located in a high-tier or low-tier RA, the radio interfaces of the mediumtier network are turned off. Moreover, when an MS is located in a low-tier or medium-tier RA, the high-tier radio interfaces are turned off. These low-tier radio interfaces are woken up periodically to check the entrance into the low-tier or middle-tier RAs [1].

\section{References}

[1] Y.W. Chumg and S.W. Lee, Modeling and performance analysis of power efficient multi-tier location management in interworked WLAN and cellular network, Journal of Mathematical and Computer Modelling 57(11-12) (January 2013), 2764-2775.

[2] Y.W. Chumg, Performance analysis of energy consumption of mobile station in three-tier network, Proceedings of the World Congress on Engineering (WCE) 2 (July 2012), 1258-1262.

[3] W. Stallings, Data and Computer Communications, Pearson Education, eighy edition, 2011.

[4] M.Ilyas and H.T. Mouftah, The Handbook of Optical Communication Networks, CRC Press LLC, first edition, 2003.

[5] Y. Fang and Y.B. Lin, Mobility management and signaling traffic analysis for multi-tier wireless mobile networks, IEEE Transactions on Vehicular Technology 54(5) (September 2005), 1843-1853.

[6] Y. Fang, Registration traffic and service availability for two-tier wireless networks, IEEE WCNC 3 (September 2000), 1090-1095.

[7] Y.B. Lin, L.F. Chang, A. Noerpel and K.I. Park, Performance modeling of multi-tier PCS system, International Journal of Wireless Information Networks 3(2) (March 1996), 67-78.

[8] Y. Agarwal, R. Chandra, A. Wolman, P. Bahl, K. Chin and R. Gupda, Wireless wakeups revisited: Energy management for VoIP over WiFi smartphones, IEEE MobiSys (2007).

[9] S.H. Tsao and E.C. Cheng, PIANO: A power saving strategy for cellular/VoWLAN dual-mode mobile wireless networks, Selected Area in Communication 14(5) (October 2008), 683-698.

[10] S.K. Lee, S.H. Seo and N. Golmie, An efficient power-saving mechanism for integration of WLAN and cellular networks, IEEE Communications Letters 9(12) (December 2005), 1052-1054.

[11] V. Gupta and S. Mohanty, Multi-radio PM draft technical requirement, IEEE 802.21 MRPM SG (December 2007).

[12] Y.W. Chung, Reducing signaling load for power-efficient integrated WLAN/cellular networks, IEICE Electronics Express 7(9) (2010), 652-658.

[13] S.K. Lee, L.Y. Kim and H.J. Kim, MIPv6-based power saving scheme in integrated WLAN and cellular networks, IEICE Transactions on Communications E90-B(10) (January 2007), 2780-2783.

[14] S.K. Lee, W.S. Chung, K.H. Hong and N. Golmie, Power-efficient communication protocol for integrated WWAN and WLAN, EURASIP Journal on Wireless Communications and Networking (November 2008), 1-13.

[15] C. Lam, Passive Optical Networks. Elsevier, first edition, 2007.

[16] R. Yadav, Passive-optical-network (PON) based converged access network [Invited], Journal of Optical Communication Network 4(11) (November 2012), B124-B130. 
[17] J. Chen et al., Scalable passive optical network architecture for reliable service delivery, Journal of Optical Communication Network 3(9) (September 2011), 667-673.

[18] Y.W. Chung, QoS-aware multi-tier location managements for integrated WLAN/UMTS networks, IFIP Networking 3976 (May 2006), 307-318.

[19] X. Lin et al., Towards understanding the fundamentals of mobility in cellular networks, IEEE Transactions on Wireless Communications 12(4) (April 2013), 1686-1698.

[20] A. Shao et al., Bandwidth allocation design to guarantee QoS of differentiated services for a novel OFDM-PON, 18th Asia-Pacific Conference on Communications (APCC) (October 2012), 775-780.

[21] X. Ye, C.M. Assi and M.A. Ali, Integrated bandwidth allocation and wavelength assignment in WDM-PON networks, IEEE Sarnoff Symposium (April 2008), 1-5.

[22] F. Aurzada et al., Capacity and delay analysis of next-generation passive optical networks (NG-PONs), IEEE Transactions On Communications 59(5) (May 2011), 1378-1388.

[23] D. Hock et al., QoS provisioning in WLAN mesh networks using dynamic bandwidth control, 14th European Wireless Conference (EW) (Jun 2008), 1-7.

[24] L. Song et al., Predictability of WLAN mobility and its effects on bandwidth provisioning, 25th IEEE International Conference on Computer Communications Proceedings (INFOCOM) (April 2006), 1-13.

[25] M.A. Marsan et al., Optimal energy savings in cellular access networks, IEEE International Conference on Communications Workshops (ICC) (June 2009), 1-5.

[26] A. Kakekhani and A.G. Rahbar, Green distributed quality of transmission aware routing and wavelength assignment in all-Optical networks, Accepted For Publication in Journal of Optical Communications (2013).

Forough Shirin Abkenar received her B.Sc. degree in Information Technology from Shahid Madany Azarbaijan university, Azarshahr, Tabriz, Iran. She is a master student in Information Technology at Sahand University of Technology, Sahand New Town, Tabriz, Iran. She is interested in cellular networks, optical networks and switching in high speed networks.

Akbar Ghaffarpour Rahbar is an Associate Professor in Electrical Engineering department at Sahand University of Technology, Sahand New Town, Tabriz, Iran. Dr. Rahbar received his B.Sc. and M.Sc. degrees in computer hardware and computer architecture both from Iran University of Science and Technology, Tehran, Iran, in 1992 and 1995 respectively. He received his Ph.D. degree in computer science from University of Ottawa, Canada in 2006. He is the director of Computer Networks Research Lab at Sahand University. Dr. Rahbar is a senior member of the IEEE. He is currently on the editorial board of Wiley Transactions on Emerging Telecommunications Technologies Journal and Journal of Convergence Information Technology. He is also the editor-in-chief of Journal of Nonlinear Systems in Electrical Engineering. His main research interests are optical networks, optical packet switching, scheduling, cellular networks, PON, IPTV, VANET, network modelling, analysis and performance evaluation, the results of which can be found in over 90 technical papers (see http://ee.sut.ac.ir/showcvdetail.aspx?id=13). 

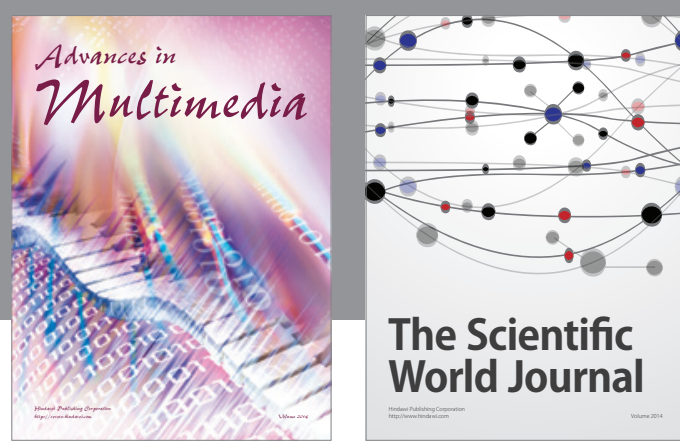

The Scientific World Journal
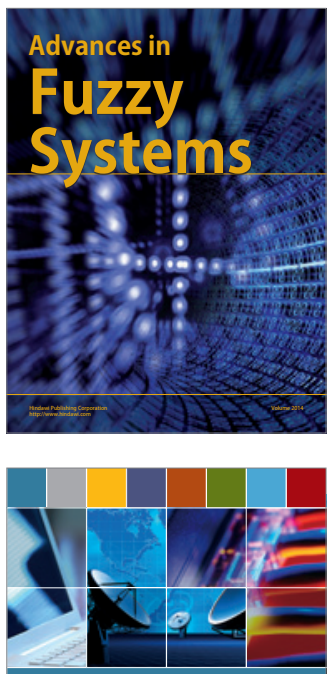

Computer Networks and Communications
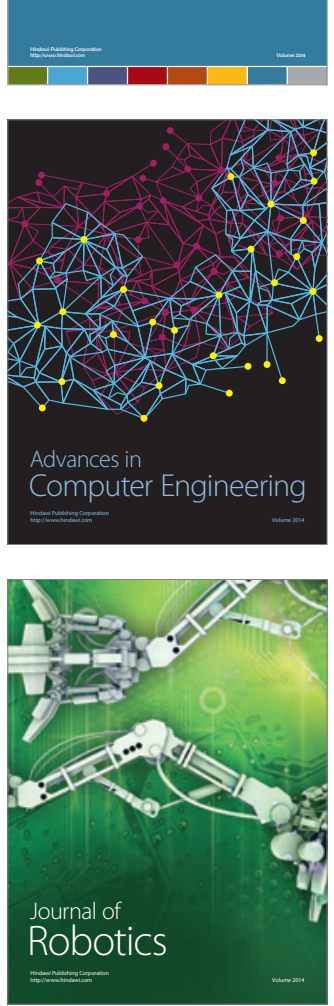
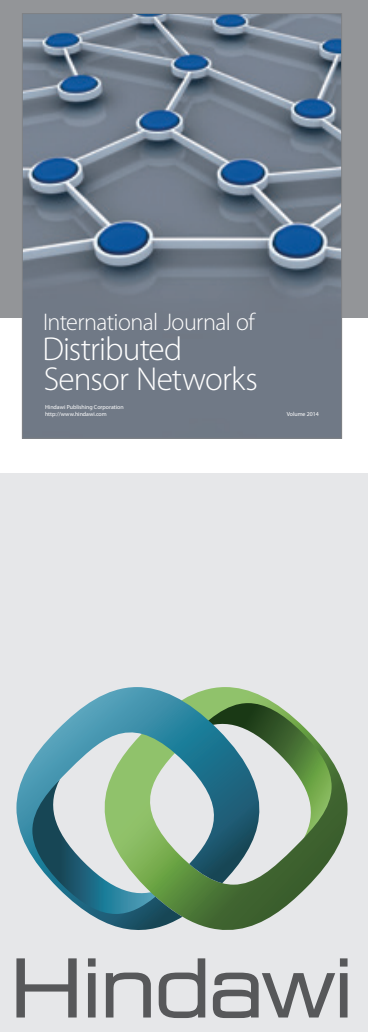

Submit your manuscripts at

http://www.hindawi.com
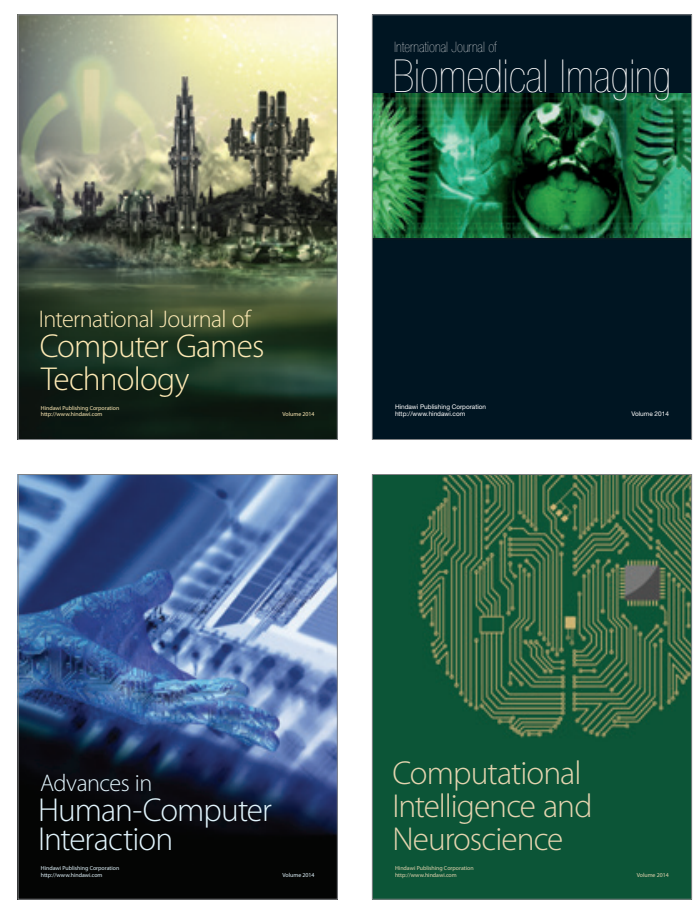
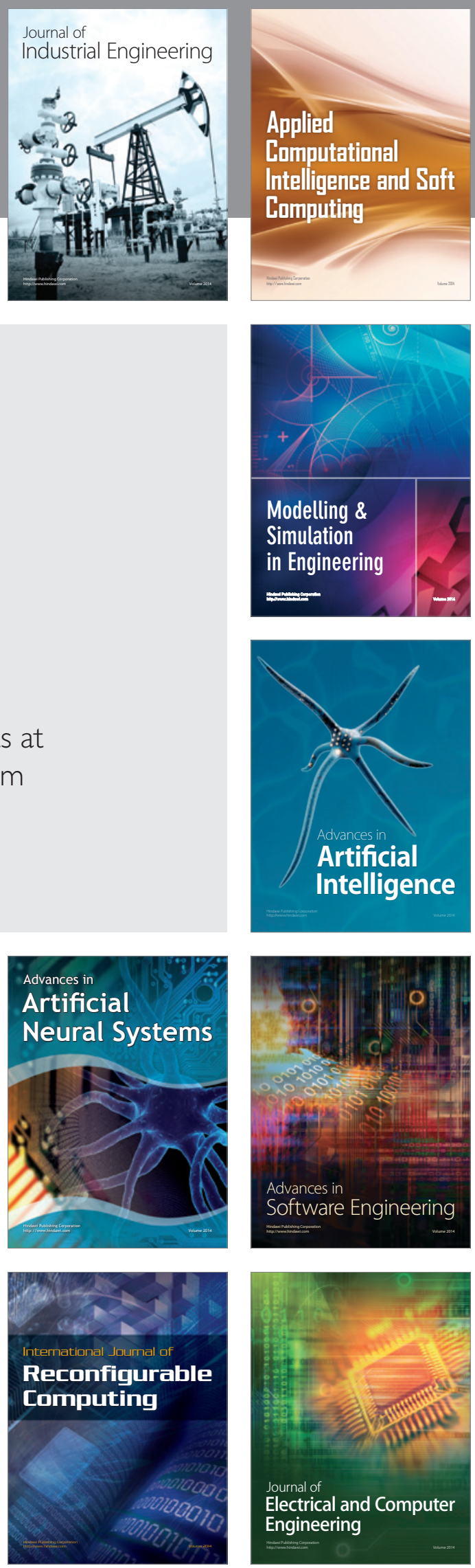